\title{
GEOLOGÍA DE LA HOJA SAN ISIDRO, COSTA RICA
}

\author{
Guillermo E. Alvarado ${ }^{1,2^{*}}$, Rafael Barquero ${ }^{1}$, Waldo Taylor ${ }^{1}$, Mauricio Mora ${ }^{2}$, \\ Giovanni Peraldo², Guillermo Salazar², Teresita Aguilar ${ }^{2}$ \\ ${ }^{1}$ Área Amenazas y Auscultación Sismológica y Volcánica, Instituto \\ Costarricense de Electricidad. Apdo. 10032-1000, San José, Costa Rica \\ ${ }^{2}$ Escuela Centroamericana de Geología, Universidad de Costa Rica \\ *Autor para contacto: galvaradoi@ice.go.cr
}

(Recibido: 12/02/08; aceptado: 20/07/09)

\section{INTRODUCCIÓN}

El área que cubre la hoja topográfica escala 1: 50000 denominada San Isidro, ubicada en el flanco pacífico de la Cordillera de Talamanca, constituida principalmente por rocas ígneas y sedimentarias, son en su mayor parte Terciarias (Fig. 1). Los depósitos cuaternarios, producto de la erosión y meteorización de las rocas más antiguas, se encuentran formando terrazas y abanicos aluviales como el Valle de San Isidro, enriquecidos en bauxita. Estos depósitos fueron motivo de acaloradas disputas al final de la década de los sesentas a raíz de una eventual explotación del aluminio por parte de una compañía privada llamada ALCOA. La bauxita, mena del aluminio, finalmente no fue explotada y los sectores más enriquecidos en este elemento fueron urbanizados, cubriendo un posible recurso mineral para futuras generaciones. Otros sectores interesantes y poco estudiados, son los prospectos de cobre (porfídico y polimetálico) en el área (Castillo, 1997).

La zona es hoy día muy visitada por tres razones fundamentales: 1) Posee la ciudad de San Isidro de El General, con un importante desarrollo socio-económico, siendo la principal ciudad de la región Brunca, 2) Es lugar de paso de la carretera Interamericana, y 3) es ruta hacia el reconocido sector de turismo ecológico y geo-glaciar del Parque Nacional Cerro Chirripó, y áreas vecinas.

No obstante, desde el punto de vista geológico, es una región tectónicamente activa y con procesos erosivos y depositacionales importantes, producto del abrupto relieve, clima lluvioso 


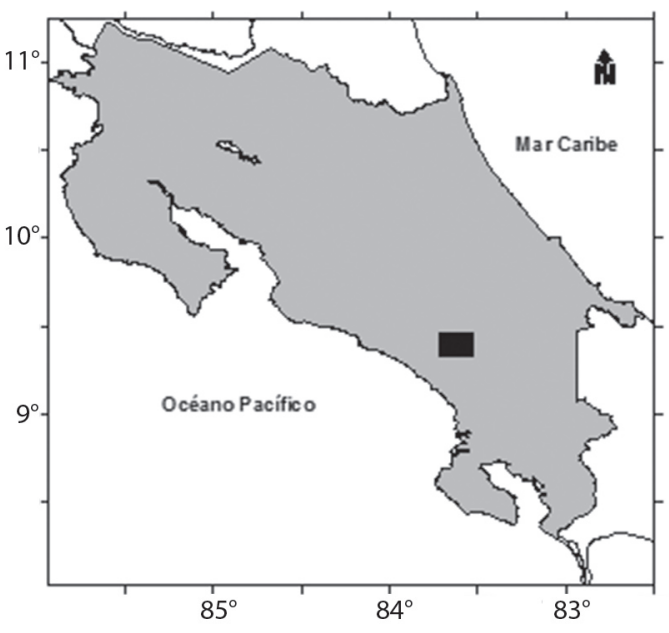

Fig. 1: Mapa de ubicación de la hoja San Isidro

y cálido, profunda meteorización de las rocas, y mal uso de la tierra. Por ello, un estudio de la geología será particularmente importante para efectos de la planificación, necesaria para una adecuada transformación del medio por parte del ser humano.

Algunas investigaciones se han realizado en el área de estudio, entre las que sobresalen los mapas geológicos compilativos de Escalante (1978), Dengo y Escalante (1980) y Mora (1983) y el levantamiento geológico parcial de la carretera Interamericana (Weyl, 1957, 1961, 1969). Estudios geológicos con cierto grado de detalle, en su mayoría a 1: 50000 y en parte son a 1: 25 000; existen los de Leandro et al. (1983), Alvarado et al. (1998), Peraldo et al. (2001). Nuestros estudios involucran la revisión de esta literatura y su control de campo en casi todos los caminos y un número importante de ríos y quebradas, realizándose modificaciones substanciales a los estudios previos. La información geológica referente al macizo del Chirripó y del Cementerio de la Máquina fue compilada en trabajo de gabinete con base en los trabajos de Calvo (1987) y Tournon y Alvarado (1997), con comprobación parcial de campo por parte del primer autor. El presente mapa es la síntesis de toda esta labor, dejándose en blanco las áreas no interpretadas o visitadas.

Uno de los mayores problemas claro está, fue la relativa pobreza de dataciones radiométricas y paleontológicas específicas, para poder correlacionar adecuadamente con las formaciones descritas en la literatura, particularmente las sedimentarias y las volcánicas.

El presente trabajo es el resultado en síntesis de una investigación geológica realizada por el ICE para el Proyecto Hidroeléctrico Chimirol, por el momento sin miras a construirse, y de dos proyectos de Investigación de la Vicerrectoría de Investigación de la Universidad de Costa Rica bajo los proyectos 113-99-239 "Geología de los ríos Chirripó y alrededores, Pérez Zeledón, Costa Rica", 113-A4-108 "Dinámica y deformación de fallas activas mediante técnicas geodésicas: implicaciones para la geodinámica externa y mitigación del riesgo sísmico en el área piloto de la falla Buenavista, distrito de Rivas, San Isidro de Pérez Zeledón” y "Estratigrafia y tectónica de áreas claves de Costa Rica", número 830-A5-047.

\section{MARCO GEOLÓGICO Y TECTÓNICO GENERAL}

La tectónica regional está caracterizada por sistemas de fallas y de fracturas producto tanto de los esfuerzos generados por el proceso de subducción de la placa del Coco bajo la placa Caribe, como por los cuerpos intrusivos que conforman la cordillera. En la zona se observan patrones de fallamiento principales de orientación NW-SE, NE-SW y NNE-SSW.

En las cercanías de San Isidro del General existe una fuente sismogénica activa que originó el terremoto de Pérez Zeledón en julio de 1983 (magnitud 6,2 $\mathrm{M}_{\mathrm{s}}$ ), el cual provocó daños importantes en casas y edificios de la zona (Boschini et al., 1988). Además, existen otros sistemas de fallas que serán tratados más adelante, que aunque no han mostrado actividad reciente, son potencialmente activos por lo que deben ser investigadas con mayor detalle.

La zona que abarca la hoja San Isidro se caracteriza por una topografía abrupta de fuertes pendientes $\left(15^{\circ}\right.$ a $\left.50^{\circ}\right)$ y cañones profundos excavados por ríos y quebradas, que se comportan como torrentes de montaña. Las montañas presen$\tan$ elevaciones que varían entre los 2000 y $3820 \mathrm{~m}$ s.n.m. y zonas bajas entre los 700 y 1300 m s.n.m. 
Dicha topografía es fiel reflejo de las condiciones litológicas propias de la cordillera de Talamanca, caracterizada principalmente por grandes cuerpos intrusivos granitoides y rocas sedimentarias, localmente metamorfizadas, así como lavas (posibles cuerpos subvolcánicos) subordinadas en la secuencia estratigráfica.

La zona es drenada por 6 ríos principales: División, Pacuar, Pedregoso, San Isidro, Buenavista y Chirripó. El río División corre hacia el SW, en tanto que los restantes drenan hacia el sur para formar el río General. Los patrones de drenaje en las partes altas, están dominados por las estructuras tectónicas principales (fallas, fracturas, planos de estratificación, etc.) originando patrones de drenaje subangulares y subparalelos. En las partes bajas, los patrones predominantes son subdentríticos. Los ríos principales muestras un perfil en $\mathrm{V}$ de su valle, debido al rejuvenecimiento por el levantamiento de la cordillera de Talamanca.

Los paleo-abanicos del Valle de El General están representados por antiguos conos de deyección con diferentes grados de disección y conservación, preferentemente ubicados a lo largo del valle del río Buenavista y en las cabeceras del río Blanco, producto de frecuentes deslizamientos activos o por la subsecuente erosión del río General, en donde se tienen los llanos interfluviales con rellenos aluviales de tributarios del General.

Referente a otras geoformas recientes, encontramos al menos cuatro diferentes niveles de terrazas aluviales y coluvio-aluviales a los largo de los ríos principales de la región (p.ej. Chirripó Pacífico), indicios de las pasados períodos de agradación/erosión aluvial por los períodos interglaciares y glaciares, así como por avenidas fluviales.

Algunos cauces abandonados logran aún observarse claramente tanto en las fotografías aéreas como en el campo (p.ej. quebrada Caño y cerca del poblado de Montecarlo). Fueron justo estos cauces a los que Protti (1996) les asignó un origen por lenguas glaciares. Sin embargo, un reconocimiento detallado en el campo de los mismos permite concluir que ambos cauces fueron originados por debris flows profundamente erosivos y no por lenguas glaciares. Lo anterior se fundamente en: a) la inexistencia de valles glaciares entre aquellos ubicados en las altas cumbres de Talamanca (sobre los $3000 \mathrm{~m}$ ) y estos cauces (ubicados a unos $1000 \mathrm{~m}$ de elevación inicial), b) los cauces efectivamente poseen bloques estriados, transportados por los debris flows desde las altas cumbres, pero nunca una roca aborregada comprobada in situ, dado que perfectamente podrían ser megalitos estriados re-depositados y cubiertos lateralmente por sedimentos. Situaciones similares se observan cerca de Rivas y Herradura. Sin embargo, más investigación es necesaria al respecto.

Las fuertes pendientes, la deforestación, el sobrepastoreo y la presencia de suelos lateríticos y areníticos, son factores que han favorecido la acción erosiva, los deslizamientos, la reptación y la remoción del escaso suelo, hecho que se vio acrecentado por el pasado terremoto de Buenavista en 1983. Además de todo lo anterior, la presencia de cuencas profundamente afectadas por un mal uso de la tierra, se comprueba en Peraldo (2004), quien reconoce desde el punto de vista de la geografía dos geocoras (unidades de paisaje): la cuenca del río Buenavista y la del rió Chirripó Pacífico, que se comportan de manera diferente a la erosión.

\section{ESTRATIGRAFÍA}

Se han reconocido en la zona siete unidades litológicas principales, que de más antigua a reciente son: A) Rocas sedimentarias: 1. Lutitas negras, areniscas y conglomerados (Fm. Térraba), 2. Lutitas y areniscas (Fm. Caraigres?), 3. Brechas volcano-sedimentarias (Fm. Pacacua), 4. Lutitas y areniscas (Fm. Peña Negra), 5. Conglomerados y areniscas aluviales y 6 . Depósitos aluviales, coluviales y glaciáricos. B) Las rocas ígneas, se dividieron en dos grandes grupos: 1) Cuerpos intrusivos (Grupo Comagmático de Talamanca), y cuerpos volcánicos (Fm. La Cruz). C) Finalmente, se tienen las cornubianitas, producto del metamorfismo de contacto.

Sin embargo, se ha de aclarar que la relación estratigráfica entre varias de estas unidades no es clara, ya sea por problemas de cobertura vegetal, suelos, ausencia de afloramientos o por ser contactos por falla. La misma distinción entre algunas rocas intrusivas básicas y lavas (sector occidental) no siempre fue fácil en el campo, lo mismo entre 
coladas de lava y cuerpos subvolcánicos, dado que existen muchos cuerpos intrusivos de grano medio así como cuerpos hipoabisales menores de grano grueso (porfiritas) a fino, que se confunden con verdaderas coladas de lavas. El grado de fracturación, meteorización y los afloramientos no ayudan a su discernimiento. Más estudios de detalle focalizados sobre este problema, son necesarios.

\section{UNIDAD DE LUTITAS NEGRAS, ARENISCAS Y CONGLOMERADOS (FM. TÉRRABA)}

Descripción: Constituida por interestratificación de areniscas finas a gruesas, claramente con una estratificación decimétrica, intercaladas con bancos de conglomerados, brechas y niveles centimétricos de lutitas. Las lutitas hasta limolitas son negras a café y constituyen estratos de espesores entre 5 y $100 \mathrm{~cm}$ o pueden presentarse masivas. Pueden ser calcáreas. Las areniscas finas, medias y gruesas, gris claro verdosas, silíceas a ligeramente calcáreas, presentan laminación paralela, cruzada, bidireccional, en algunos casos presentan fuertes variaciones laterales en el espesor. Los granos son subredondeados a redondeados, moderadamente clasificados. Los conglomerados hasta brechas son verdosos, con fuerte influencia volcánica, evidenciada por la composición de sus clastos. Se presentan como bancos y rellenos de canal. También se determinó la presencia de niveles enriquecidos en troncos fósiles y trozos de carbón (río Pacuar). Fragmentos fósiles casuales de bivalvos fueron observados en grandes bloques al lado del camino.

Localidad: Los principales afloramientos se localizan a lo largo del río Pacuar.

Aspectos regionales: Forma parte de la secuencia sedimentaria que se extiende a lo largo de toda la Fila Costeña. El espesor máximo aflorante comprobado de esta unidad supera los $1000 \mathrm{~m}$, dado que nunca se llega a observar su base ni su techo en el área estudiada, ya que este último está limitado por falla y cubierto por depósitos más recientes.
Relaciones estratigráficas: Aunque nunca se llegó a observar directamente en el campo el contacto entre esta unidad con la Unidad de lutitas y areniscas, se infiere que las infrayace, tal y como se desprende del mapa geológico.

Edad geológica: Los microfósiles plantónicos cercanos al techo (tajo cercano a 366,0492,0), tales como Globigerina praebulloides praebulloides, G. ciperoensis ciperoensis, G. ampliapertura y G. opima opima, nos indican una edad de Oligoceno Medio temprano (acrozona concurrente, Fig. 2), claramente correlacionable en edad, litología y por cercanía geográfica con la Formación Térraba, ampliamente extendida hacia Dominical y Palmar.

Aspectos específicos: La presencia de estratos negros medianamente estratificados y con pocas estructuras sedimentarias y pobres en fósiles, sugiere un ambiente relativamente "tranquilo" y algo anóxico, de quizás plataforma abierta que rápidamente varió a más somero dada la influencia del continente evidenciado por la presencia de troncos de madera y trazas de carbón, así como canales conglomerádicos y estructuras de corrientes bidireccionales.

\section{UNIDAD DE LUTITAS Y ARENISCAS (FM. CARAIGRES?)}

Descripción: Constituida por interestratificaciones de lutitas y areniscas (areniscas y graywackes líticas), con laminación paralela, cruzada, bidireccional, posibles briozooarios y globigerinoides, con fuerte influencia volcánica (clastos de rocas volcánicas: 3-70\%, mayoritariamente alrededor de un 60\%).

Localidad: Los principales afloramientos se presentan en los alrededores de San Ramón Norte y Sur.

Aspectos regionales: Forma parte de la secuencia sedimentaria que se extiende a lo largo de toda la Fila Costeña.

Relaciones estratigráficas: Aunque nunca se llegó a observar directamente en el campo, se infiere que sobreyace a la unidad de Lutitas Negras, areniscas y conglomerados 


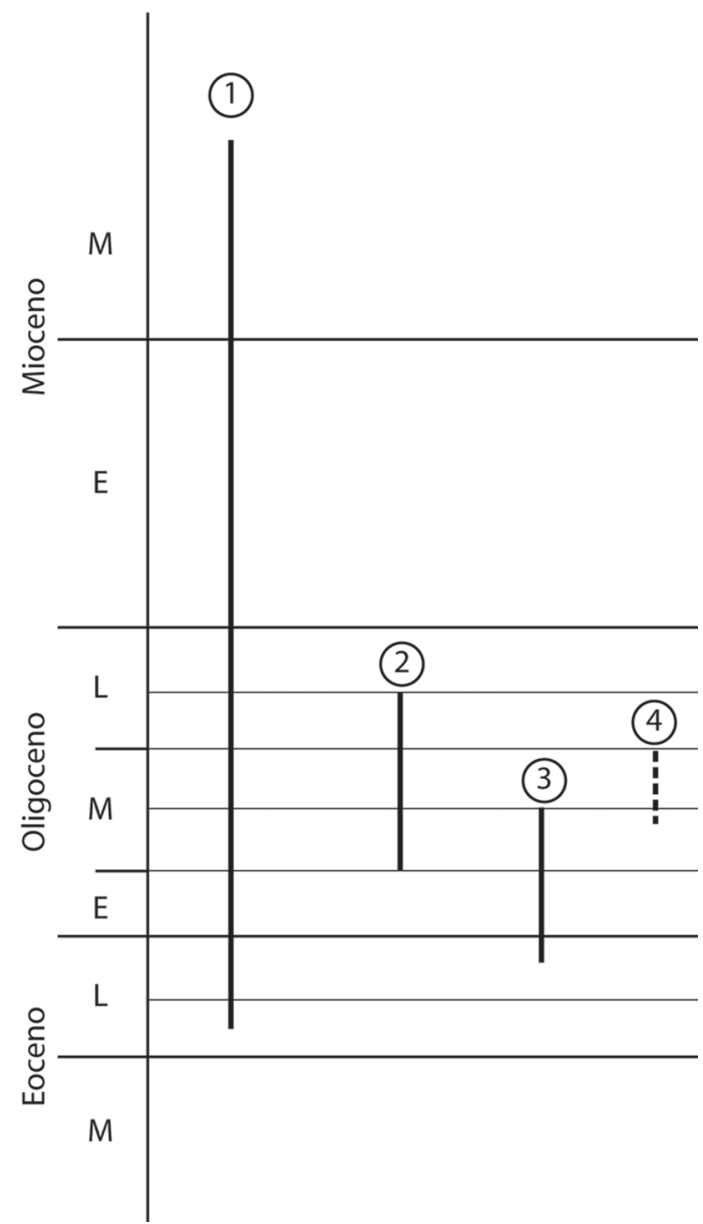

Fig. 2: Rangos de edades de los microfósiles de Térraba en el área de estudio.

previamente descrita, y subyace a la de Brechas volcano-sedimentarias.

Edad geológica: Dada su posición estratigráfica y litología, se puede asignar una edad Oligocena Media a Superior.

Aspectos específicos: Algunas facies parecen ser de ambiente profundo, tipo turbidítico, tales como aquellas presentes en el camino que comunica San Ramón Norte de Pérez Zeledón con Savegre (377,98-491,65). Se encontraron icnofósiles del tipo Chondrites isp., indicadores de un ambiente bajo en oxígeno, generalmente del talud continental.

\section{UNIDAD DE VOLCANO-SEDIMENTARIA (FM. PACACUA)}

Descripción: La secuencia está conformada por lutitas, areniscas (graywackes líticas) y brechas, verdosas y rojizas a moradas, así como con niveles enriquecidos en pisolitos, constituyendo verdaderas tobas de lapilli acrecional (p.ej. Tajo Chimirol, 503,4 - 376,6), generalmente al tope de las secciones observadas. Las brechas son rocas duras cuando están sanas, poco o nada estratificadas, gris-violáceas o verdosas, con clastos angulares a subangulares de origen volcánico (andesitas, algunas escorias) en una matriz de grano fino (contacto flotante o soporte por granos) constituida por feldespatos y piríboles cloritizados. Su granulometría varía desde bloques decimétricos (unos $25 \mathrm{~cm}$ de diámetro) en contacto puntual hasta fragmentos centimétri$\cos (25-70 \%)$, subangulares a subredondeados, en una matriz volcarenítica. También pueden presentarse como brechas de grano fino (pocos centímetros a milímetros de diámetro). Dentro de las brechas, en la fila Helechal se localizó una ignimbrita epidotizada. Una descripción de esta secuencia es mostrada por Peraldo et al. (2001).

Localidades: Se les observa entre las poblaciones de Rivas y Guadalupe, incluyendo el Tajo Chimirol camino a San José de Rivas. Se pueden observar en algunos cortes recientes de la carretera interamericana (entre las coordenadas 494,6 378,0 a 494, 5-372,0) generalmente asociada a los deslizamientos rotacionales de ruptura profunda.

Aspectos regionales: Su litología indica un vulcanismo cercano. Algunas brechas podrían ser en su mayoría brechas intrasedimentarias por intrusiones de cuerpos hipoabisales en sedimentos (peperitas); otras son tobitas de lapilli (volcano-ruditas).

Relaciones estratigráficas: $\mathrm{Su}$ espesor es difícil de estimar, pudiéndose conjeturar un espesor mínimo de $260 \mathrm{~m}$. Por su posición estratigráfica y litología, se correlaciona con la Formación Pacacua, descrita por Denyer y Arias (1991) para el Valle Central.

Edad geológica: En vista que las rocas presumiblemente sobreyacentes son intruidas por los 
granitoides de Talamanca (12-8,5 Ma), ésta correspondería teóricamente con la edad mínima de dichas rocas, por lo que se podría especular una edad Oligocena Superior a Miocena Inferior para los eventos volcánicos y sedimentarios que les dieron origen.

Aspectos específicos: La presencia de esta unidad correlacionable con la Fm. Pacacua, nos habla de una volcano-sedimentación extendida desde el actual Valle Central hasta este sector de la Zona Sur de Costa Rica, tal y como lo sugiere el Mapa Geológico de Costa Rica (Denyer y Alvarado, 2007).

\section{UNIDAD DE LUTITAS Y ARENISCAS (FM. PEÑA NEGRA)}

Descripción: Constituida por interestratificaciones de lutitas, limonitas y areniscas. Las lutitas hasta limolitas son negras a café y constituyen estratos de espesores entre 5 y $15 \mathrm{~cm}$ o pueden presentarse masivas con pirita; pueden ser calcáreas. Las areniscas finas, medias y gruesas, gris claro verdosas, silíceas, presentan laminación paralela y cruzada, y en algunos casos presentan fuertes variaciones laterales en el espesor, con acuñamiento lateral de las capas, contactos ondulados. Los granos son subredondeados a redondeados, moderadamente clasificados. El espesor varía entre 5 y $80 \mathrm{~cm}$, y generalmente se presentan muy fracturadas. Se observa la estratificación y laminación original, con estratos tabulares de 5 a 40 $\mathrm{cm}$ de espesor que contienen pirita y pátinas de manganeso. El espesor máximo aflorante de esta unidad es de unos $800 \mathrm{~m}$.

Localidades: Los principales afloramientos se localizan en el camino a San Ramón Sur, Santa Eduviges, Carretera Interamericana, Pueblo Nuevo y camino a Palmital, Rivas, Chimirol y Canaán.

Aspectos regionales: Forman una faja de rocas sedimentarias fuertemente afectadas por rocas volcánicas, hipoabizales e intrusivas (principalmente gabroides) aunado a las fallas del pie de la cordillera de Talamanca. Todo ello complica el esquema estratigráfico.
Relaciones estratigráficas: Aunque nunca se llegó a observar directamente en el campo el contacto entre esta unidad con la Unidad volcano-sedimentaria, se infiere que la sobreyace concordantemente. Dada su posición estratigráfica y litología, se puede correlacionar con la Formación Peña Negra, descrita por Denyer y Arias (1991) para el Valle Central.

Edad geológica: Fósiles casuales de bivalvos se han llegado a observar in situ, o como niveles fosilíferos enriquecidos en forma de cantos aluviales en los ríos. Se observaron fósiles casuales $(502,75-378,50$ y 504,35-376,60) de la clase Bivalva Propeamusium (Parvamusum) sp. Indet., Echinodermata del género Clypeaster sp. (espinas y placas de erizos irregulares, observadas cerca de San José de Rivas en 504,35376,60), y de la clase Rizopoda representados foraminíferos planctónicos preservados como moldes. El ambiente del género Propeamusium es de aguas profundas (especies actuales viven a más de $2000 \mathrm{~m}$ ), y su rango de edad fósil varía del Eoceno-Oligoceno (Panamá) al Mioceno Medio en la Fm. Peña Negra (Peraldo et al., 2001).

Por su posición estratigráfica, litología, los fósiles descritos, y dado que están intruidas por los granitoides de Talamanca (12-8,5 Ma), edad mínima de dichas rocas, por lo que podría especular una edad Miocena Inferior a Media Temprana.

Aspectos específicos: La presencia de estratos negros medianamente estratificados y con pocas estructuras sedimentarias, y pobre en fósiles, sugiere un ambiente relativamente tranquilo y anóxico.

\section{CONGLOMERADOS Y ARENISCAS FLUVIALES (FM. VALLE DE EL GENERAL)}

Antecedentes: Dóndoli (1943) la define como "Terrazas compuestas por materiales aluvionales en su mayoría de origen volcánico". Henningsen (1965) hace también mención de la presencia de abanicos aluviales en el flanco pacífico de la cordillera de Talamanca. Castillo (1978) la define por primera vez como Formación Valle de El General y estudia estas áreas desde el 
punto de vista económico para la explotación de sus depósitos bauxíticos. Madrigal (1977) identifica que algunos de ellos han sido basculados por efectos tectónicos del Cuaternario. Dicha unidad fue posteriormente descrita por Alán (1983). No obstante, Kesel (1983) amplio el ámbito de cobertura de los depósitos que componen a esta unidad, a la cual le sumó los de la Unidad Brujo de Mora (1979), redenominando ambas (Valle de El General y Brujo) como Formación Brujo, dándose una evidente confusión.

Descripción: Esta unidad está representada por abanicos de deyección aluvial formados por fanglomerados de guijarros y bloques de rocas ígneas de hasta $4 \mathrm{~m}$ de diámetro, flotantes hasta en contacto puntual en una matriz de arcilla y arena (Mora, 1979; Alán, 1983). El alto grado de laterización bauxítica que muestra en algunos sectores ha ocasionado el interés por la explotación de aluminio (Alán, 1983). Kesel (1983) ha reportado sobretodo materia orgánica (troncos, plancton, hojas).

Localidades: Los mejor desarrollados del área son el de Buenos Aires, Platanares, Helechales, Sábalo y Blanco (Mora, 1979). Su espesor máximo estimado es de 400 m (Mora, 1979) hasta $600 \mathrm{~m}$ (Kesel, 1983).

Aspectos regionales: Se trata de abanicos coalescentes que se presentan desde San Isidro de El General hasta por lo menos Buenos Aires.

Relaciones estratigráficas: Según Mora (1979), los abanicos aluviales de la Formación Valle de El General sobreyacen parcialmente y en forma posiblemente discordante a la mayoría de las unidades previamente descritas y solo es sobreyacida por los depósitos recientes.

Edad geológica: Kesel (1983) asigna una edad Pliocena-Cuaternaria a esta formación, siendo su edad más reciente verificada con base en dataciones de carbono 14.

Aspectos específicos: Los abanicos están juxtapuestos y son coalescentes. Su origen es el producto de la destrucción erosiva de la cordillera de Talamanca durante su emplazamiento y por el deshielo de glaciares pleistocénicos. A causa de ello, es posible distinguir varias generaciones de abanicos y se nota que por lo general su tamaño disminuye con la juventud relativa (Mora, 1979).

\section{DEPÓSITOS ALUVIALES, COLUVIALES Y GLACIÁRICOS}

Descripción: Se trata especialmente de bloques métricos de rocas intrusivas y gran cantidad de aluviones de medios a gruesos, provenientes de las estribaciones de la Cordillera de Talamanca.

Localidades: Se observan especialmente en los ríos Buenavista, Chirripó Pacífico, General, Quebradas y Pedregoso. Frente a la población de Canaán, por el drenaje del río que desciende del cerro Chucuyo, se presenta un abanico de bloques y detritos ígneos, incluyendo arenas, producto de un deslizamiento que ocurrió en la década de los setentas del siglo pasado.

Relaciones estratigráficas: Sobreyacen a todas las unidades pues son los materiales más recientes.

Edad geológica: Cuaternario.

\section{ROCAS ÍGNEAS}

\section{CUERPOS VOLCÁNICOS (FM. LA CRUZ)}

Descripción: Está constituida por lavas de tipo andesítico hasta basáltico y brechas. Las lavas son de color gris oscuro a negro con textura afanítica-porfirítica, fenocristales de plagioclasa y piroxenos. Son rocas densas con fracturamientos de tipo prismático. Muchos de ellos podrían corresponder con cuerpos hipoabisales (probablemente ante la ausencia de brechas de progresión) múltiples, y menos probablemente de coladas de lava, aunque en su mayor parte, los afloramientos no permitieron una mejor definición.

Localidades: Las lavas y brechas afloran principalmente en las partes altas como Alto Jaular y cerro Chinga, en Rivas, Pueblo Nuevo y Santa Eduviges afloran principalmente las brechas. Un afloramiento notorio es La Piedra o el Cristo, que resguarda al Valle El General.

Edad geológica: Dos dataciones radiométricas específicas para la hoja San Isidro se presentan (Jackson, 1991; de Boer et al., 1995): una para un basalto olivínico por La Ese $(21,65 \pm$ 
$1,08 \mathrm{Ma}$ ) y otra para una dacita por Las Juntas (se desconoce si se trata de un bloque: $11,79 \pm 0,59$ Ma), abarcando un rango del Mioceno Inferior al Medio. La mayoría de las lavas y cuerpos hipoabisales con textura lávica datados en el Cerro de la Muerte, aportan edades entre 10,50 y 13,00 Ma (MacMillan et al., 2004), es decir más antiguas y coetáneas con las intrusiones y en parte con los sedimentos.

Relaciones estratigráficas: Los contactos no fueron observados con claridad de modo que se desconoce si están intercaladas dentro de la secuencia sedimentaria, si son cuerpos hipoabisales o si están sobreyaciendo discordantemente. Se les asocia, sin embargo, con la Formación La Cruz. Algunas lavas parece que subyacen a las Brechas volcanosedimentarias, situación que parece estar clara en la secuencia estratigráfica del río Blanco $(503,4$ 374,0 a 504,0 - 373,2, ubicación aproximada).

Antecedentes: Ya Malavassi et al. (1971) y MacMillan et al. (2004) dejan entrever la existencia de un vulcanismo (coladas de lava y cuerpos hipoabisales) intercalados e intruyendo respectivamente, a una secuencia de sedimentos marinos coetáneos.

\section{CUERPOS INTRUSIVOS (GRUPO COMAGMÁTICO DE TALAMANCA)}

Antecedentes: En escritos de W. Gabb del siglo XIX se hace referencia a las rocas intrusivas de Talamanca (Gabb, 1875). La construcción de la carretera Interamericana en la parte norte de la cordillera y las ascensiones a los diversos cerros de dicha cordillera, puso en evidencia la diversidad petrográfica de esos conjuntos intrusivos. En efecto, Weyl $(1957,1961,1969)$ menciona rocas y cuerpos plutónicos de tamaño muy variable en la Cordillera de Talamanca que intruyeron a las series sedimentarias, lavas y tobas del Eoceno hasta el Mioceno Inferior. Idem (1969) incluye análisis químicos de muestras en la carretera Interamericana Sur. Dengo (1962) comenta algunos aspectos de la "Serie Comagmática de Talamanca", mientras que Berrangé y Whittaker (1977) introdujeron el nombre "Grupo Comagmático (intrusivo) de Talamanca".
Kussmaul (1987) hace una síntesis sobre la petrología de las rocas intrusivas Neógenas de Costa Rica y propone el nombre "Grupo Granito-Gabro de Talamanca".

Descripción: Las rocas en el área predominantes corresponden con gabros (gabros cuarzosos con anfíbol, gabros cuarzoso con clinopiroxeno uralitizado y gabro leucocrático con augita), y monzogabros cuarzosos con clinopiroxeno uralitizado (p. ej. Catarata del río Blanco: 383,26-505,65), o monzonitas cuarzosas con anfíbol cloritizado con transición a granito y granito leucocrático (puente frente al Templo católico de Herradura: 381,40-505,55). Una diorita con biotita se observa en la localidad de Herradura $(380,45-507,30)$. Un dique gabróico leucrático intruye a los sedimentos morados de Pacacua (501,19-376,37). Inclusiones comagmáticas, brechas magmáticas y los microdiques también están presentes. Las rocas más leucocráticas se presentan hacia el núcleo de los batolitos, mientras que hacia la periferia y cuerpos menores (stocks) suelen ser melanocráticos.

Localidades: En la literatura no se ha definido ninguna localidad tipo. Excelentes afloramientos se observan en la ruta al parque Nacional Cerro Chirripó.

Aspectos regionales: $\mathrm{El}$ Intrusivo de Talamanca forma stocks y batolitos de diversa forma y extensión, en su mayoría con un eje aproximadamente orientado NW-SE. Los más extensos afloramientos ocurren en el eje más elevado de la cordillera, en superficies superiores a los $100 \mathrm{~km}^{2}$ y desniveles de hasta $2000 \mathrm{~m}$, como por ejemplo en los macizos de los cerros Chirripó, de la Muerte, Dúrika, Kamuk (Tournon y Alvarado, 1997).

Relaciones estratigráficas: Estas rocas intruyen principalmente a las lavas y rocas sedimentarias. La cornubianitas se encuentran tanto en posición lateral como superior.

Edad geológica: Para el área de estudio parecen existir algunas dataciones que nos indican un rango entre 9,8 y 7,8 Ma (de Boer et al., 1995; MacMillan et al., 2004). Datos K/Ar y ${ }^{40} \mathrm{Ar} /{ }^{39} \mathrm{Ar}$, en especial sobre los macizos calco-alcalinos de Tapantí, Cerro de la Muerte, División y Kamuk, indican edades entre 12 y 7,8 m.a. (Berrangé y Whittaker, 1977; Bellon y Tournon, 1978; Appel, 1990; de Boer et al., 1995; MacMillan 
et al., 2004), es decir en su mayoría Mioceno Superior.

Aspectos específicos: La perturbación tectónica es mínima, existiendo incluso estratificación horizontal en las capas sedimentarias intruidas, lo que evidencia velocidad constante y uniformemente distribuida de los cuerpos plutónicos. Las texturas, así como el tipo de las rocas encajantes, sugieren un emplazamiento en niveles estructurales superficiales (Tournon y Alvarado, 1987).

\section{CORNUBIANITAS}

Descripción: Los sedimentos metamorfizados (cornubianitas) poseen tonos gris claros a oscuros, endurecidos y suelen presentar pirita (p.ej. en el sector de Monterrey) y pátinas de manganeso, además de cloritización, silicificación, sericitización, epidotización, carbonatización y matriz filomórfica, producto de una alteración hidrotermal incipiente. Se presentan como un halo alrededor de las intrusiones y en relación con la roca caja (generalmente sedimentarias).

Localidad: Los principales afloramientos se localizan cerca de las cabeceras de los ríos Chirripó Pacífico y Buenavista, así como a lo largo de ciertos tramos de la carretera Interamericana.

Aspectos regionales: Las intrusiones provocaron en las rocas caja diferentes tipos de alteraciones y metamorfismo. Cerca de los contactos con las rocas sedimentarias, éstos han sido transformados en cornubianitas. Weyl (1957) describe una epidotización de las lavas y tobas: frecuentemente se encuentra una transformación parcial de los minerales primarios a epidota, pero también se observan vetillas de epidota y cuarzo o hasta una epidotización total, como en el tajo $\mathrm{km} 121$ de la carretera Interamericana Sur o en las faldas del cerro Chirripó. Otros tipos de alteración hidrotermal son piritización (Cañón del Guarco y km 92,5), seritización (Tajo Fuentes), alteración potásica a biotita y feldespatos potásicos secundarios y por último unas aureolas muy anchas de alteración propilítica (epidota, cuarzo, calcita, clorita) (Kussmaul, 1987).

Relaciones estratigráficas: Se presenta al contacto visible o no con los cuerpos intrusivos.
Edad geológica: Contemporánea con las intrusiones.

\section{GEOLOGÍA ESTRUCTURAL Y NEOTECTÓNICA}

Con base en el análisis de fotos aéreas y observaciones de campo se determinaron 3 sistemas principales de fallamiento (Leandro et al., 1983; Alvarado et al., 1998; Denyer et al., 2003):

Sistema N-S: Se deduce por el trazado rectilíneo de los cauces de los ríos San Ramón, Quebradas, Buena Vista y parte del río Blanco.

Sistema NE-SW: Controla el curso de los ríos y quebradas División y Páramo y la quebrada Blanca, entre otras.

Sistema NW-SE: Al igual que el sistema anterior controla el curso de muchos ríos y quebradas como por ejemplo los ríos Chimirol, Blanco y quebradas Boquete, Aristides y Seca. La falla Aristides-Quebrada Seca fue comprobada en el campo con un cambio litológico a ambos lados de las quebradas y una zona de falla de $2 \mathrm{~m}$ en la carretera Interamericana.

Diaclasas: En general las rocas aflorantes en la zona presentan gran cantidad de diaclasas originadas probablemente por el emplazamiento del plutón de Talamanca o por cambios térmicos. En las rocas intrusivas los patrones de diaclasamiento presentan dos direcciones predominantes de $\mathrm{N} 20^{\circ} \mathrm{W}-\mathrm{N} 05^{\circ} \mathrm{W}$ y $\mathrm{N} 05^{\circ} \mathrm{W}-\mathrm{N} 20^{\circ} \mathrm{E}$ con ángulos de inclinación entre $40^{\circ}$ y $90^{\circ}$. Las fracturas presentan superficies lisas y son en general abiertas.

Los sedimentos aluviales (terrazas y abanicos) de la Formación Valle de El General, no muestran indicios fotogeológicos ni en el campo de grandes desplazamiento tectónicos, al menos en los recorridos realizados durante la corta investigación de campo.

En la localidad de Herradura existen aguas termales $(380,45-507,30)$ en una serraría ubicada al oriente del río Blanco, asociadas con un sistema de falla $\mathrm{N} 32^{\circ} \mathrm{E} ; 85^{\circ} \mathrm{NW}$.

Dóndoli et al. (1968) sugieren la probable existencia de un pliegue sinclinal a lo largo del Valle de El General. Sin embargo, fue Madrigal (1977) quién describe por primera vez con cierto 
grado de detalle, que los abanicos aluviales sobre la Fila Costeña presentan cierto plegamiento (basculamiento o inclinación) contrario a su pendiente original de depositación, sugiriendo que algunos de ellos han sido perturbados por efectos tectónicos del Cuaternario, a modo de una estructura monoclinal (en realidad él quiso decir homoclinal, es decir una estructura con un buzamiento extendido en un único sentido). No obstante, deberá de evaluarse en futuras investigaciones si se trata de una estructura sinclinal, dado que la contraparte en los flancos de Talamanca corresponde más bien con el ángulo de depositación original de los abanicos provenientes. Lo importante es que en el sector de los abanicos, existen dataciones radiométricas que indican edades entre más de 37000 y unos 8800 años (Kesel, 1983). Por lo menos tres edades comprendidas entre 17000 y 8000 años, si parecen estar efectivamente afectadas por el basculamiento.

\section{AGRADECIMIENTOS}

Varias personas colaboraron en diferentes momentos en diferente grado, entre ellos los colegas Allan López y Gerardo Soto, los asistentes Francisco Arias, Raúl Rodríguez, Ronald Monge, Jorge Quesada, Rigoberto Torres, German Arce, Fernando Quesada, Rosa Vázquez, Ronald Brenes, Luis Madrigal y María Ester Torres, todos ellos del ICE.

Los resultados del presente trabajo se nutrieron en su última etapa del proyecto de investigación 113-A4-108 "Dinámica y deformación de fallas activas mediante técnicas geodésicas: implicaciones para la geodinámica externa y mitigación del riesgo sísmico en el área piloto de la falla Buenavista, distrito de Rivas, San Isidro de Pérez Zeledón" de la Vicerrectoría de Investigación de la Universidad de Costa Rica, a través del Programa Institucional de Investigación en Desastres No. 605-A3-952, así como "Estratigrafía y tectónica de áreas claves de Costa Rica", número 830-A5047, y del Instituto Panamericano de Geografía e Historia (IPGH) mediante su proyecto GEOF 3.4.2.51.

\section{REFERENCIAS}

ALÁN, M.A., 1983: Geología y estudios de lateritas en el extremo noroeste del Valle de El General. Costa Rica.- 123 págs. Univ. Costa Rica.[Tesis Lic.]

ALVARADO, G.E., BARQUERO, R. \& TAYLOR, W. 1998: Informe geológico del P.H. Chimirol para la etapa de Estudios Preliminares.- 15 págs. [Inf. interno ICE].

APPEL, H. 1990: Geochemie und K/ArDatierung an Magmatiten in Costa Rica. Zentralamerika.- 149 págs. Univ. Mainz, Alemania [Tesis Ph.D.]

BERRANGÉ, J.P. \& WHITTAKER, J.E., 1977: Reconnaissance Geology of Tapanti quadrangle, Talamanca Cordillera, Costa Rica.- Inst. Of Geological Overseas Div., British Museum rep. 37: 136-142.

BELLON, H. \& TOURNON, J., 1978: Contribution de la géochronométrie K-Ar a l'étude du magmatisme de Costa Rica et América Central.- Bull. Soc. Geol. de France. 20(6): 955-959.

BOSCHINI, I., ALVARADO, G.E. \& ROJAS, W., 1988: El terremoto de Buenavista de Pérez Zeledón (julio 3, 1989): Evidencia de una fuente sismogénica intraplaca desconocida en Costa Rica.- Rev. Geol. Amér. Central, 8: 111-121.

CALVO, G., 1987: Geología del macizo del Chirripó, Cordillera de Talamanca, Costa Rica.- 37 págs. Escuela Centroamericana de Geología, Univ. Costa Rica [Inf. Campaña Geológica].

CASTILLO, R., 1978: Geología regional preliminar de una parte del Valle de El General, Costa Rica.- Departamento de Desarrollo Geológico y Recursos Minerales, CODESA, Bol. Geol., 1: 289-293. 
CASTILLO, R., 1997: Recursos Minerales de Costa Rica. Génesis, distribución y potencial.- 221 págs. Ed. Univ. Costa Rica.

DE BOER, J.Z., DRUMMOND, M.S., BORDELON, M.J., DEFANT, M.J., BELLON, H. \& MAURY, R.C., 1995: Cenozoic magmatic phases of the Costa Rican island arc (Cordillera de Talamanca).- En: P. MANN (ed), Geologic and Tectonic Development of the Caribbean Plate Boundary in Southern Central America.- Spec. Pap., Geol. Soc. Amer. Special Paper, 295: 35-55.

DENGO, G., 1962: Tectonic-igneous sequence in Costa Rica.- En: Petrologic Studies. A.F. Buddington volume: 133-161. -Geol. Soc. Amer., 132-160.

DENGO, G. \& ESCALANTE, G., 1980: Mapa preliminar de reconocimiento geológico y fotogeológico. Zona de Influencia de la Cuenca del río Savegre, San José [Inf. interno ICE].

DENYER, P. \& ARIAS, O., 1991: Estratigrafía de la región Central de Costa Rica.- Rev. Geol. Amér. Central, 12: 1-59.

DENYER, P. \& ALVARADO, G.E., 2007: Mapa Geológico de Costa Rica.- escala 1: 450 000. Librería Francesa S.A.,

DENYER, P. MONTERO, W. \& ALVARADO, G.E., 2003: Atlas tectónico de Costa Rica.81 págs. Ed. Univ. Costa Rica, San José.

DÓNDOLI, C., 1943: La Región del General: Condiciones geológicas y geoagronómicas de la zona. -Dept. Nacional de Agricultura, Instituto de Defensa del Café, Tomo XIII, $\mathrm{N}^{\circ} 106: 513-528$.

DÓNDOLI, C., DENGO, G. \& MALAVASSI, E., 1968: Mapa Geológico de Costa Rica.Escala 1: 700 000. Ministerio de Industria y Comercio, Dirección de Geología, Minas y Petróleo.
ESCALANTE, G., 1978: Mapa geológico preliminar del área suroeste de Costa Rica (Cordillera de Talamanca y zonas adyacentes).- Escala 1: 200 000.- En: CASTILLO, R., Geología de Costa Rica: una sinópsis, Editorial UCR.

GABB, W.M., 1875: Notes on Costa Rica Geology. Amer.- Tour. Sci., 9: 198-204.

HENNINGSEN, D. 1965: La fila costeña del Pacífico de Costa Rica y su posición dentro del sistema montañoso centroamericano meridional. 90 págs. Edición especial. Dir. Gral. Geol. Min. Petrol. Ministerio de Industria y Comercio. Costa Rica.

JACKSON, T.E., 1991: Neogene geochemistry of the Central American Arc: Western Panama and Southern Costa Rica.- 109 págs. Univ. South Florida [Tesis MSc.]

KESEL, R.H., 1983: Quaternary History of the Río General Valle, Costa Rica.- Nat. Geogr. Res. Rep. 15: 339-358.

KUSSMAUL, S. 1987: Petrología de las rocas intrusivas neógenas de Costa Rica.- Rev. Geológica de Amér. Central, 7: 83-111.

LEANDRO, G, LEÓN, C., AVILA, M., FERNÁNDEZ, A., ELIZONDO, J., MONTALTO, F., CHÁVEZ, R., \& OBANDO, J., 1983: Informe geológico-sismológico San Isidro de Pérez Zeledón y alrededores.- 56 págs. [Inf interno ICE].

MACMILLAN, I., GANS, P.B. \& ALVARADO, G., 2004: Middle Miocene to present plate tectonic history of the southern Central American Volcanic Arc.Tectonics, 392: 325-348.

MADRIGAL, R. 1977: Evidencias geomórficas de movimientos tectónicos recientes en el valle El General.- Ciencia y Tecnología, 1 (1): 97-108. 
MALAVASSI, E., LAMBERT, A. \& WEYL, R., 1971: Excursión a Talamanca.- 36 págs. Trabajo Inédito, febrero 1971.

MORA, S., 1979: Estudio geológico de una parte de la región sureste del Valle de El General, Provincia de Puntarenas, Costa Rica.- 188 págs. Univ. de Costa Rica[Tesis Lic.]

MORA, S., 1983: Mapa de estructuras tectónicas del Pacífico Central de Costa Rica.- escala 1: 200 000 Dept. Geología, Instituto Costarricense de Electricidad [Informe interno].

PERALDO, G., SALAZAR, L. G., AGUILAR, T., 2001: Estudio Geológico del valle medio del Río Chirripó Pacífico y alrededores, Pérez Zeledón, Costa Rica.- 38 págs. Informe final, proyecto 113-99-239, Universidad de Costa Rica.

PERALDO, G., 2004: La novedad de lo constante: El proceso del desastre y su relación con la Gestión Territorial, caso de los distritos de Rivas y General, Pérez Zeledón,
Costa Rica.- 194 págs. Universidad de Costa Rica [Tesis MSc,].

PROTTI, R., 1996: Evidencias de glaciación en el Valle del General (Costa Rica) durante el Pleistoceno Tardío.- Rev. Geol. Amér. Central, 19/20: 75-85.

TOURNON, J. \& ALVARADO, G.E., 1997: Carte géologique du Costa Rica: notice explicative.- 80 págs y mapa escala 1500000 . Ed. Tecnológica de Costa Rica.

WEYL, R., 1957: Contribución a la Geología de la cordillera de Talamanca de Costa Rica, Centro América. San José, Costa Rica.- Min. Obras Públicas. Inst. Geogr. de Costa Rica.

WEYL, R., 1961: Die Geologie Mettelamerikas.226 págs. Borntraeger, Berlin.

WEYL, R., 1969: Magmatische Förderphasen und Gesteinschemismus in Costa Rica (Mittlelamerika).- N. Jl. Geol. Paläont. Mh, 7: 423-446. 